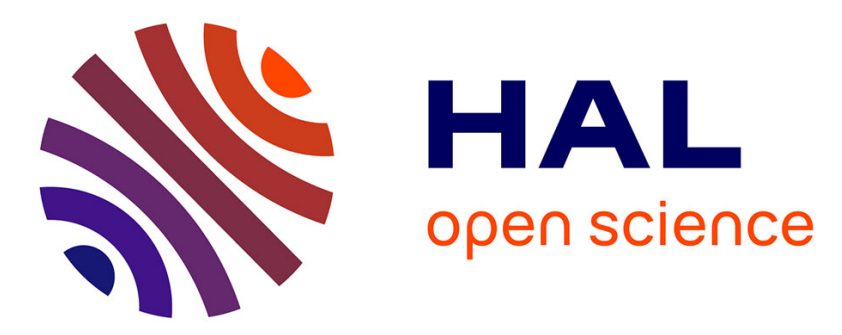

\title{
Réinterprétation sociale locale et mémoires familiales de l'immigration aveyronnaise à Pigüé (Province de Buenos Aires, Argentine)
}

\author{
Christophe Albaladejo, Susana Sassone, Roberto Bustos Cara
}

\section{To cite this version:}

Christophe Albaladejo, Susana Sassone, Roberto Bustos Cara. Réinterprétation sociale locale et mémoires familiales de l'immigration aveyronnaise à Pigüé (Province de Buenos Aires, Argentine). Autrepart - Revue de sciences sociales au Sud, 2017, 2017/4 (84), pp.13-31. hal-02338161

\section{HAL Id: hal-02338161 \\ https://hal.science/hal-02338161}

Submitted on 29 Oct 2019

HAL is a multi-disciplinary open access archive for the deposit and dissemination of scientific research documents, whether they are published or not. The documents may come from teaching and research institutions in France or abroad, or from public or private research centers.
L'archive ouverte pluridisciplinaire HAL, est destinée au dépôt et à la diffusion de documents scientifiques de niveau recherche, publiés ou non, émanant des établissements d'enseignement et de recherche français ou étrangers, des laboratoires publics ou privés. 


\title{
RÉINTERPRÉTATION SOCIALE LOCALE ET MÉMOIRES FAMILIALES DE L'IMMIGRATION AVEYRONNAISE À PIGÜÉ (PROVINCE DE BUENOS AIRES, ARGENTINE)
}

\author{
Christophe Albaladejo, Susana Sassone et Roberto Bustos Cara
}

Presses de Sciences Po | «Autrepart »

$2017 / 4 \mathrm{~N}^{\circ} 84$ | pages 13 à 31

ISSN 1278-3986

ISBN 9782724635379

Article disponible en ligne à l'adresse :

https://www.cairn.inforevue-autrepart-2017-4-page-13.htm

Distribution électronique Cairn.info pour Presses de Sciences Po.

(C) Presses de Sciences Po. Tous droits réservés pour tous pays.

La reproduction ou représentation de cet article, notamment par photocopie, n'est autorisée que dans les limites des conditions générales d'utilisation du site ou, le cas échéant, des conditions générales de la licence souscrite par votre établissement. Toute autre reproduction ou représentation, en tout ou partie, sous quelque forme et de quelque manière que ce soit, est interdite sauf accord préalable et écrit de l'éditeur, en dehors des cas prévus par la législation en vigueur en France. Il est précisé que son stockage dans une base de données est également interdit. 


\section{Réinterprétation sociale locale et mémoires familiales de l'immigration aveyronnaise à Pigüé (Province de Buenos Aires, Argentine)}

Christophe Albaladejo*, Susana Sassone**, Roberto Bustos Cara***

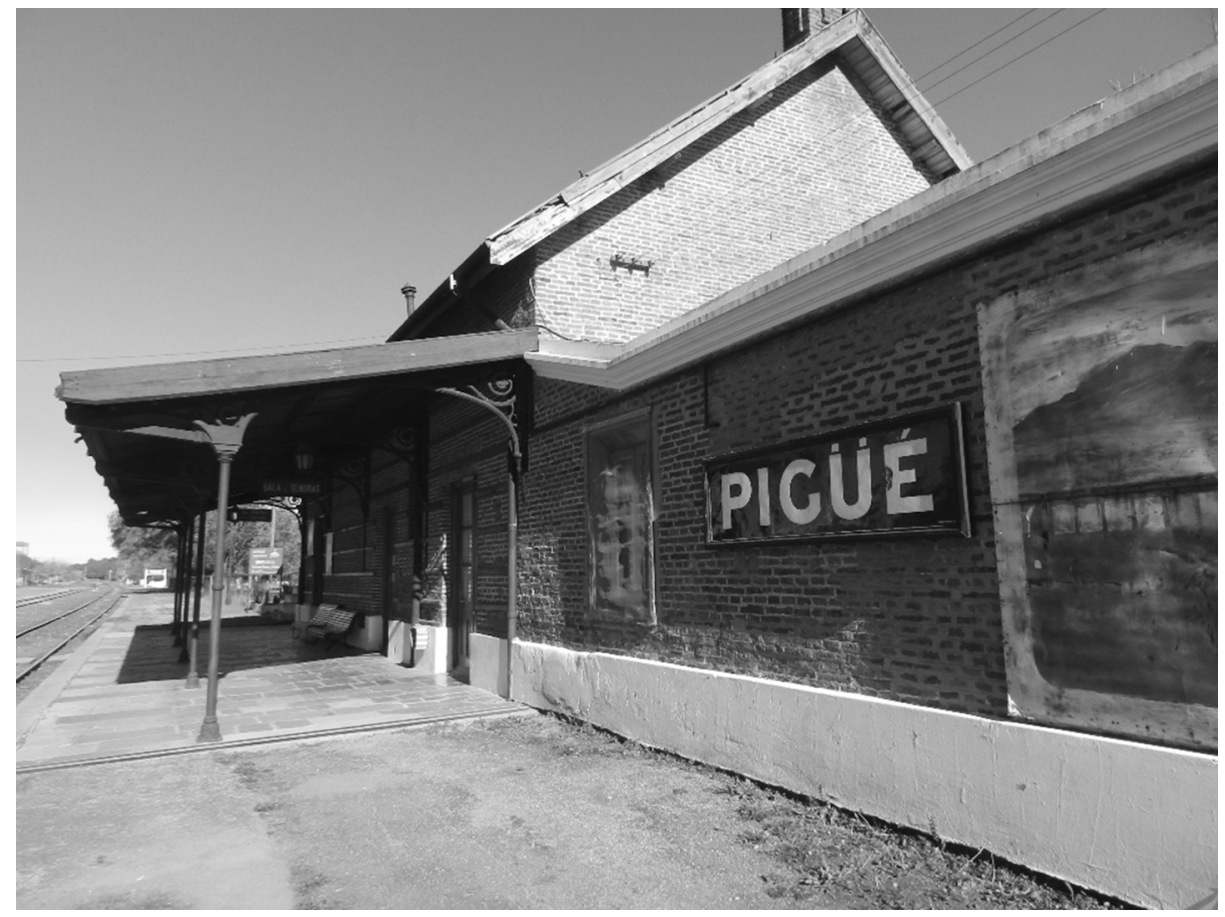

La gare ferroviaire de Pigüé, construite juste avant leur arrivée, a accueilli le premier contingent d'immigrants aveyronais en 1884, photographie de Susana Sassone.

* Géographe, Agriterris/Inra et Conicet/Imhicihu, Buenos Aires, Argentine.

** Géographe, Conicet/Imhicihu, Buenos Aires, Argentine.

*** Géographe, Agriterris/Universidad Nacional del Sur, departamento de geografía y turismo, Bahía Blanca, Argentine. 
Bien que cet article aborde l'héritage français dans la géographie spécifique d'une localité, il est important de clarifier que les Français ont aussi, après les Espagnols et les Italiens, marqué l'identité argentine. Les recherches des historiens en rendent compte, comme le montre la bibliographie exhaustive de Rey Balmaceda [1994] sur l'immigration, la colonisation et les collectivités étrangères en Argentine, à travers une centaine de références à des livres, des articles scientifiques ou journalistiques et des rapports inédits sur les migrants français en Argentine. La lecture des titres de ces sources fait ressortir l'installation française sous la forme de colonies agricoles dans la pampa argentine. De son côté, Otero [2012] a écrit la première histoire des Français en Argentine dans un ouvrage mobilisant les sources argentines et françaises. Son travail fait également ressortir le rôle de la colonisation agricole, et la capacité des migrants français à créer des associations culturelles et des écoles pour la reconnaissance et le maintien de la culture et de la langue française. Cet auteur décrit ce processus tant pour le milieu urbain que pour les villages de la pampa où se sont installées des familles françaises, dont Pigüé. Cette dernière est une petite ville de 17000 habitants, chef-lieu du district de Saavedra de 3,500 $\mathrm{km}^{2}$, comportant également quatre villages de moins de 3000 habitants, et située à $560 \mathrm{~km}$ au sud-ouest de la ville de Buenos Aires (figure 1). Elle est connue dans toute l'Argentine pour sa colonisation française, mais aussi dans l'Aveyron, car les familles des colons provenaient de ce département de la France.

Figure 1 - Situation de Pigüé et des villages du district de Saavedra
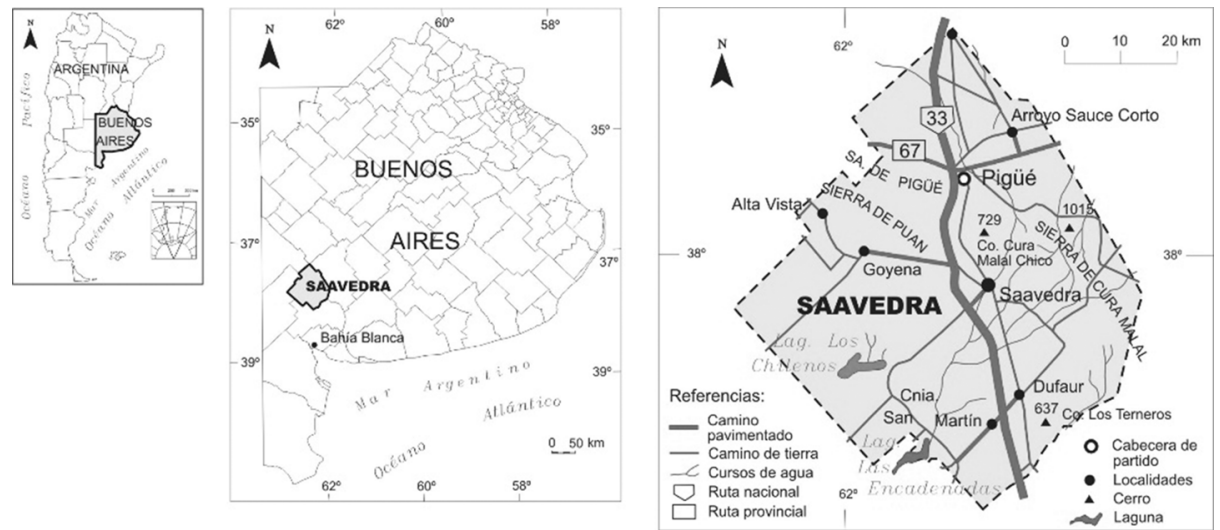

Source : Laboratoire de cartographie du département de géographie et tourisme, Universidad Nacional del Sur, Bahía Blanca, Argentine.

Ce passé français n'a pas toujours été reconnu ni même tout simplement connu, pas seulement en Aveyron ou en Argentine, mais aussi au plan local par ses habitants et les acteurs locaux. Il est le résultat d'une prise de conscience ou d'une redécouverte pour certains, voire d'une découverte pour bien d'autres, au cours d'un processus de construction d'une mémoire sociale (familiale et collective) et donc d'appropriation collective de ce passé de colonisation et du lien avec 
l'Aveyron, et aussi plus largement, de la culture française. Pourtant les habitants de Pigüé d'origine française sont aujourd'hui une minorité. Comment un habitant de Pigüé, qu'il soit ou non d'origine française, peut-il se sentir concerné par cette culture et comment se l'approprie-t-il depuis ses propres pratiques socioculturelles et son identité ? Nous proposons dans cet article d'analyser la transformation du passé français, d'un bien privé en un bien qui n'est pas seulement « collectif», mais public, autrement dit à notre sens, fondateur de la collectivité territoriale locale.

Lavabre [2007, p. 147] attire l'attention sur la polysémie de la notion de « mémoire » et sur le fait qu'elle embrasse beaucoup de choses différentes (souvenirs, commémorations, monuments, etc.). Selon Baussant [2007, p. 289], cette notion n'engage pas uniquement le recouvrement du souvenir, mais aussi les processus dynamiques de relecture des représentations sociales collectives, et donc la construction des identités présentes, ainsi que des souvenirs eux-mêmes. Les travaux fondateurs de la sociologie de la mémoire sont ceux de Maurice Halbwachs [1925] qui montre comment l'individu construit ses souvenirs en s'aidant des cadres de la mémoire sociale. À la fin de sa vie, Halbwachs renforce le caractère actif et construit de la notion de mémoire, mettant en évidence l'importance des représentations, des aspirations, mais aussi des données factuelles du présent dans la reconstruction individuelle et sociale des souvenirs [1950]. Pour Halbwachs, le passé n'est pas conservé, il est constamment construit à partir du présent [Lavabre, 2000]. Il faut attendre les années 1970 pour que se développe la sociologie de la mémoire et que cette notion se fasse reconnaître aux côtés de celles de culture, d'identité, de passé, de souvenir. Un peu plus tard, cette sociologie croise l'analyse des migrations qui sont devenues un phénomène majeur dans nos sociétés contemporaines. De fait, Baussant montre comment nos sociétés, sous l'effet de la mondialisation, se saisissent de la mémoire dans ce qui prend des allures de «courses à l'historicisation et à la patrimonialisation » [Baussant et al., 2015, p. 12]. Dans cet ensemble théorique faisant une large place aux interprétations des acteurs et au contexte social présent, nous avons réalisé des entretiens semi-directifs de 90 à 120 minutes centrés sur les récits des acteurs à propos de leur participation à la valorisation de la culture française. Aucun entretien n'a été répété dans le cadre de cette recherche sur la mémoire sociale du lien à la France à laquelle nous avons consacré un dispositif spécifique en 2016 et 2017. Par contre, un certain nombre de nos interlocuteurs avaient déjà été enquêtés entre 1982 et 2015, mais sur un autre sujet portant sur le développement local et les liens entre la petite ville rurale et l'activité agricole. Ce sont au total 19 entretiens qui ont été réalisés et enregistrés sur les lieux de travail ou de résidence des enquêtés (sauf dans un cas où il a été effectué au salon de notre hôtel), en présence d'aucune autre personne. La première partie consistait en un récit libre de leur histoire personnelle dans ses relations à la culture française. La seconde partie de l'entretien, moins intimiste, a systématiquement pris la forme d'une conversation centrée sur leur vision de la place de la culture française au plan local. Quatre autres entretiens ont été réalisés en France en 2017 avec les acteurs de la coopération avec Pigüé. 
Tableau 1 - Liste des 23 entretiens réalisés en mars 2016 et mai 2017 en Argentine et en France

\begin{tabular}{|c|c|c|}
\hline Types d'acteurs & $\begin{array}{c}\text { Nombre } \\
\text { d'entretiens }\end{array}$ & Acteurs \\
\hline Institutions territoriales locales & 3 & $\begin{array}{l}\text { Secrétaire à la culture de la } \\
\text { municipalité } \\
\text { Conseillers municipaux } \\
\text { Directrice du Musée }\end{array}$ \\
\hline $\begin{array}{l}\text { Familles d'origine française et } \\
\text { responsables de la Société française }\end{array}$ & 5 & $\begin{array}{l}\text { Familles Issaly, Ayrinhac, } \\
\text { Marcenac, Champredonde. }\end{array}$ \\
\hline $\begin{array}{l}\text { Institutions à Pigüé pour la } \\
\text { coopération avec la France }\end{array}$ & 3 & Amicale et Alliance française \\
\hline $\begin{array}{l}\text { Bénéficiaires de la coopération } \\
\text { avec la France qui ne sont pas } \\
\text { d'origine française }\end{array}$ & 3 & $\begin{array}{l}\text { Anciens boursiers ou bénéficiaires } \\
\text { d'échanges de courte durée }\end{array}$ \\
\hline $\begin{array}{l}\text { Représentants des autres } \\
\text { communautés à Pigüé }\end{array}$ & 4 & $\begin{array}{l}\text { Société espagnole et Société } \\
\text { italienne } \\
\text { Un représentant de la communauté } \\
\text { des Allemands de la Volga } \\
\text { Un moine orthodoxe }\end{array}$ \\
\hline $\begin{array}{l}\text { Responsables aveyronnais de la } \\
\text { coopération française, entretiens } \\
\text { réalisés en France }\end{array}$ & 4 & $\begin{array}{l}\text { Association Rouergue-Pigüé } \\
\text { Conseil général de l'Aveyron }\end{array}$ \\
\hline
\end{tabular}

Source : élaboration par les auteurs.

Le traitement des entretiens s'est inspiré de l'analyse structurale du récit [Demazière, Dubar, 1997], afin de mettre en relief la logique de chacun d'entre eux, puis nous avons recherché les grands thèmes qui traversaient l'ensemble des récits. L'analyse a mis en évidence deux processus qui ne sont pas portés par les mêmes sujets et institutions : une patrimonialisation du lien avec la France fondée sur la famille et l'identité individuelle, et une patrimonialisation de ce lien construit sur l'appartenance au territoire local et une appropriation par ses habitants d'un passé de migrants qui permet à un habitant de Pigüé non descendant de Français de percevoir que le patrimoine de l'immigration aveyronnaise lui appartient également.

Nous allons dans un premier temps passer en revue le processus de construction historique des acteurs, pour aborder l'analyse de leurs stratégies dans une deuxième partie, tandis que la dernière partie sera consacrée à la construction de traces reconnues socialement. 


\section{L'immigration française en Argentine}

La politique de promotion de l'immigration par l'État argentin était inscrite dès 1853 dans la Constitution nationale. Les actions ont été diverses : mise en place d'agences de recrutement et en Espagne, en Italie et en France ; distribution de billets subventionnés et création de colonies publiques ou privées [Devoto, 2003]. Le premier recensement de 1869 comptait 32326 Français, puis 94098 dans le recensement suivant en 1895. À cette période, Otero [2012, p. 15] indique qu'en Argentine presque 3 habitants sur 100 étaient nés en France. Ce chiffre était assez semblable à celui des Espagnols en 1869, mais ces derniers étaient 198685 en 1895, soit deux fois plus que les Français. L'écart s'est encore creusé par la suite. En 1914, qui est l'année qui comptabilise le taux d'étrangers le plus élevé parmi la population argentine (30\%), le nombre de Français n'était plus que de 80 570. De l'avis des auteurs, il s'agit d'une collectivité atypique qui a eu une influence considérable par son apport culturel, au-delà de son importance numérique, si on la compare à la collectivité allemande. L'Argentine est, il est vrai, le pays d'Amérique latine qui comporte le plus grand nombre de Français et, dans cet ensemble, Pigüé est la plus importante contribution française à la colonisation agricole en Argentine.

\section{De la colonisation aveyronnaise de Pigüé à la construction sociale de la mémoire}

Le processus de transformation de la relation avec la culture française d'un bien privé (familial) en un bien public (local) a commencé par la célébration du centenaire de la fondation de Pigüé (1984) mais, pour le comprendre, il faut restituer cet événement dans une histoire longue.

En 1880, Eduardo Casey (investisseur d'origine irlandaise) acheta 275000 hectares et obtint que le chemin de fer passe sur ses terres à condition toutefois de fonder trois colonies, dont Pigüé. Il signa un contrat avec Clément Cabanettes pour le charger d'organiser cette colonisation qui, à son tour, recruta son ami Issaly pour aller chercher des colons dans leur Aveyron natal, touché de plein fouet par la crise du phylloxéra. Le 4 décembre 1884, selon les récits historiques publiés [Ducos, 1934 ; Gaignard, 1989], un contingent de 40 familles, soit 162 personnes, provenant directement de leurs villages du nord de l'Aveyron, descend à la station de train dénommée Pigüé pour s'y installer. Les seules infrastructures existantes étaient la gare, ainsi qu'un grand hangar destiné à entreposer les futures récoltes et qui servit de logement de fortune les premières semaines. Et pourtant, Cabanettes, l'entrepreneur de la colonisation, lui aussi aveyronnais, avait promis de fournir aux familles des abris convenables sur leurs lots de terre de 100 hectares chacun. Vita, la petite fille de François Issaly - l'Aveyronnais qui recrutât ces familles dans leurs villages et les accompagna dans leur voyage jusqu'à sa mort, 50 ans plus tard, et dans leurs aventures d'agriculteurs du Nouveau monde - a lu ces débuts de la colonisation dans le journal de son grand-père. Selon elle, les 
difficultés extrêmes des premières années d'installation dans une plaine hostile, pratiquement sans aide de Cabanettes ni du propriétaire des terres, ont fortement soudé cette première communauté et renforcé la reconnaissance de François Issaly comme leur leader.

Ce premier contingent de 1884 fut bientôt rejoint par un second de 120 familles en 1888, issues des mêmes villages, mais disposant d'un petit capital de départ. Celles-ci bénéficièrent des aménagements collectifs réalisés par les tout premiers colons, puis par des immigrés de diverses nationalités, dont des Français d'autres régions, des Espagnols, des Italiens, des Allemands de la Volga, etc. Aujourd'hui, la collectivité d'origine française est largement minoritaire en termes démographiques, mais elle a été à l'origine de la fondation des lieux (en particulier par le premier contingent) et elle a fourni une grande partie des notables agricoles locaux (c'est surtout le cas pour le second contingent). Depuis 1984, année du centenaire de la fondation de la ville, le passé français a pris une place dominante dans l'écriture collective de l'histoire locale du territoire. Nous allons analyser les principaux événements de la communauté française de Pigüé et de la mise en patrimoine local de cette relation avec ce passé.

Selon l'analyse de nos entretiens, appuyés sur les données des récits historiques publiés [Andreu et al., 1993; Ducos, 1934], nous proposons de distinguer trois grandes périodes dans le processus de patrimonialisation (figure 2). Les 50 premières années sont celles d'un fonctionnement communautaire, voire familial, de la culture française. La référence à la France semble avoir été d'abord celle à l'Aveyron natal et être basée sur la famille en tant que pratique sociale quotidienne, ainsi que sur des souvenirs privés et récents. Il ressort de nos entretiens que, dans l'intimité des échanges, la référence linguistique était davantage l'occitan (appelé le «patois ») que le français. Cependant, cette dernière était la langue cultivée et des écrits, et elle était mieux maîtrisée par les migrants du second contingent, non pas tellement du fait d'une date plus tardive d'émigration (quatre années seulement les séparent), mais du fait d'un statut socioculturel un peu supérieur. C'est le temps des pionniers et de la fondation de leurs institutions, comme des coopératives, qui sont parmi les premières fondées en Amérique du Sud, notamment la coopérative d'assurance contre les risques du climat sur les récoltes «El Progreso », en 1898. La plus ancienne est la Société française de secours mutuels, créée en 1891, afin de subvenir aux frais de santé et à tous les types d'imprévus touchant la communauté française de Pigüé. Il est significatif qu'en 1909, le Gouvernement de la province de Buenos Aires obligeât la société française, pour être en accord avec la loi, à rédiger ses statuts en espagnol, et non plus exclusivement en français, et à annuler la clause qui restreignait les nouvelles adhésions aux ressortissants de nationalité française ou à leurs descendants. Aujourd'hui, le rôle de la «Sociedad francesa » est symbolique, mais reste centré sur la famille et un lien généalogique à la France. De fait, elle organise, pour un cercle privé, des célébrations d'événements qui, dans un autre contexte, seraient incontestablement de nature publique, comme c'est le cas du 14 juillet. 
Figure 2-Chronologie de l'institutionnalisation du passé français à Pigüé

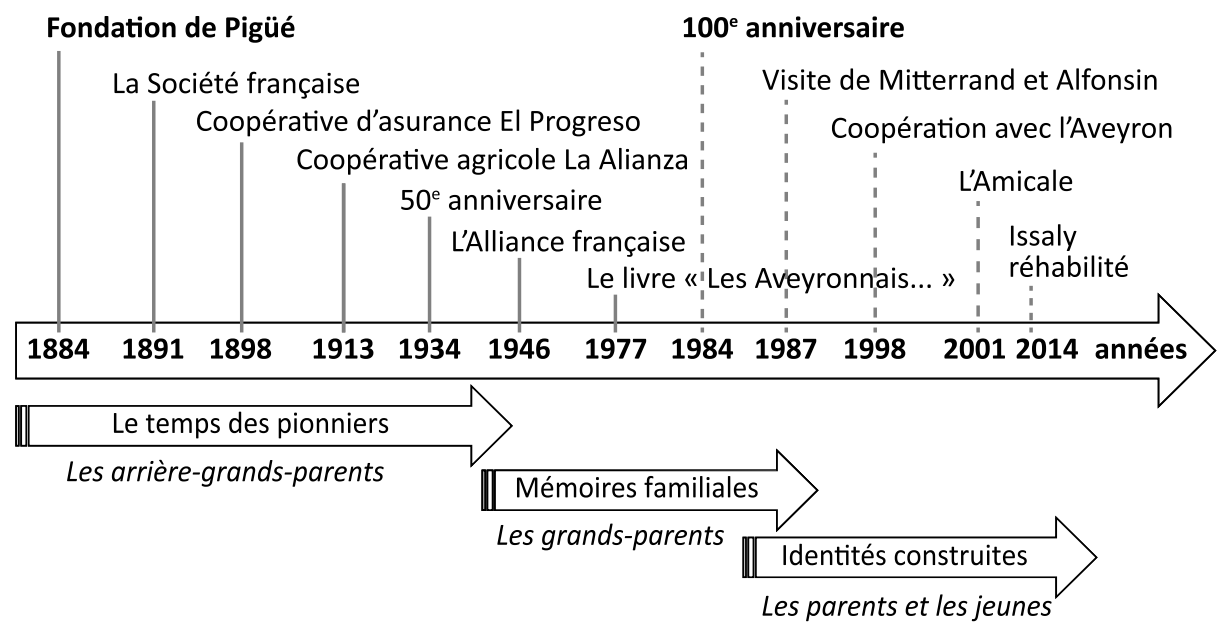

Source : Élaboration par les auteurs.

La célébration du cinquantième anniversaire de la fondation a donné lieu à un important événement public et aux premiers efforts de mise en patrimoine du passé de la colonisation française, comme en témoigne la publication pour cette occasion du premier livre d'histoire locale écrite par Octavio Federico Ducos (1934-1936) et du livre autobiographique d'Anaïs Viala [Di Liscia, Lassalle, 2002]. Ducos est un grand propriétaire terrien et éleveur, originaire de la région de Pau, qui a fourni une histoire officielle héroïque et pacifiée de la fondation de Pigüé par la colonisation française ; Anaïs Viala est une femme aveyronnaise du premier groupe fondateur de migrants, de condition très modeste, arrivée en 1884, qui a écrit dans son journal intime un témoignage terrifiant sur la condition des femmes rurales de son époque. Cet anniversaire marque ainsi le début d'une période de construction locale de la mémoire au-delà du cercle familial et donc d'une mémoire collective. L'installation à Pigüé, en 1946, d'une antenne de l'Alliance française montre l'attachement au maintien de la langue «des origines », mais aussi l'incapacité à la perpétuer dans le cercle familial.

Le début de cette période, qui est celle de la troisième génération, est marqué par des visites remarquables comme celle du premier président de la République argentine, après le retour de la démocratie à la fête du centenaire et, trois ans plus tard, en compagnie de son homologue français François Mitterrand. Les contextes nationaux ont aussi eu leur importance, en particulier le retour à la démocratie de l'Argentine en 1984, mais aussi la décentralisation de la France mise en œuvre dès 1982 sous le gouvernement socialiste. La visite des élus aveyronnais, en 1984, a abouti quelques années plus tard, dès 1998, à la mise en place d'un fructueux programme de coopération décentralisée entre la province de Buenos Aires et la région Midi-Pyrénées, en particulier avec le conseil général de l'Aveyron. Les 
chevilles ouvrières de cette coopération ont été, en France, l'Association Rouergue-Pigüé et, à Pigüé, une nouvelle association créée en 2001, l'Amicale, qui est aujourd'hui un des principaux acteurs de la patrimonialisation du lien avec la culture française.

Cette coopération binationale a permis de nombreux échanges, notamment scientifiques, avec le financement de projets de recherche, de voyages d'études et de bourses de thèse ou de master, de publications d'ouvrages [Andreu et al., 1993 ; Issaly, 2014 ; Litre Valetin, 2009 ; Samson, Bécouez, 2013 ; Tulet, Albaladejo, Bustos Cara, 2001]. Ces subventions ont été accordées à des descendants de Français, mais pas seulement. Beaucoup plus que de simples commémorations ou une exaltation du souvenir, il s'agit, à notre sens, de la construction d'un nouveau lien entre Pigüé et la France, sur la base de la mise en patrimoine collective.

\section{Les acteurs de la patrimonialisation et les stratégies complémentaires du souvenir : I'identité comme héritage ou comme construction}

Nous pouvons distinguer trois groupes d'acteurs aux stratégies patrimoniales singulières. Le premier est constitué des descendants de migrants d'origine française qui cherchent à entretenir la mémoire familiale de leurs liens avec la France et l'Aveyron. Le deuxième est composé de descendants de Français sans mémoire des origines et d'habitants du district sans lien généalogique avec la France, mais possédant une expérience récente avec ce pays (souvent à travers les programmes de coopération scientifique mentionnés ci-avant) qui cherchent à (re)construire un lien et à le développer. Enfin, le troisième groupe inclut des acteurs privés et publics, des particuliers et des collectivités, intéressés par une valorisation économique ou symbolique des liens entre Pigüé et la France.

Dans les faits, le premier groupe d'acteurs (les descendants des immigrés français) est relativement limité numériquement, car il implique d'avoir un lien généalogique avec la France et, plus précisément, avec les colons aveyronnais. Or, ce lien généalogique est certes nécessaire, mais il n'est pas suffisant. En effet, beaucoup d'habitants de Piguié ayant perdu la mémoire de leur passé familial ne se sont jamais rapprochés de la société française. Ceux qui ont maintenu cet ancrage mémoriel ont mis en œuvre des stratégies pour l'entretenir en construisant un capital symbolique reconnaissable. La maîtrise de la langue française, voire occitane, et son utilisation au sein de la famille en est un exemple. De manière générale, on observe qu'à Pigüé, la plupart des descendants d'immigrés français ayant conservé un lien avec leur origine étrangère sont issus de ou entretiennent une proximité sociale avec les notables agricoles locaux.

Le deuxième groupe d'acteurs (les descendants de Français qui redécouvrent leurs origines et les habitants de Pigüé souhaitant développer un lien avec la France) émerge dès 1984, à partir de la mise en place d'échanges académiques 
avec la France. Il se renforce après 1998, grâce aux programmes de coopération décentralisée. En Argentine, l'acteur principal de cette dynamique est l'Amicale Pigüé, créée en 2001, pour promouvoir les échanges avec l'Aveyron. Cette association a été portée par des personnes qui souhaitaient « redécouvrir leur passé français oublié » et par des acteurs publics français de la coopération (le conseil général de l'Aveyron et son bras associatif Aveyron international) qui firent immédiatement de l'Amicale Pigüé un interlocuteur privilégié. L'objectif affiché était explicitement de partager la culture française avec les habitants de Pigüé, mais également du district de Saavedra, qu'ils soient ou non d'origine française.

Le passé français est conçu et présenté comme un bien commun et collectif, une caractéristique du territoire local. Cette construction d'un «être ensemble » d'origine française fondateur du territoire est continuellement mise en débat. Elle est parfois controversée, notamment lorsqu'il s'agit d'octroyer des subventions (bourses et voyages d'étude) à des personnes ne possédant pas de lien généalogique avec la France. Lorsque les bénéficiaires n'ont pas de lien avec la France, ces expériences ont créé des liens forts et durables, parfois sur deux générations lorsque ces séjours ont impliqué l'installation en France de toute la famille (parents et enfants) sur des périodes prolongées. L'Amicale Pigüé et ses alliés de la coopération en France ont ainsi suivi une stratégie d'ouverture à tous les habitants du district, pour le partage de la culture et des appartenances en lien avec la France.

La mise en patrimoine du lien avec la France s'accompagne ici de la mise en place d'une nouvelle relation ou d'un renouvellement de ce lien. Souvent, les membres de l'Amicale Pigüé n'avaient pas conservé la mémoire de leurs origines françaises. On observe que la trajectoire de ces familles s'inscrit dans des difficultés économiques et qu'elles possèdent un statut socioculturel inférieur à celui des acteurs du premier groupe. Il est possible que cette dévalorisation sociale ait contribué à ne pas valoriser un passé de migration et à en effacer le souvenir. Nous avons notamment noté qu'une partie importante des membres de l'Amicale Pigüé sont les descendants du premier contingent de colons aveyronnais; à l'inverse, les acteurs du premier groupe (notables aisés aux origines françaises revendiquées) sont plutôt issus de la deuxième vague de migrants aveyronnais.

Dans le monde contemporain globalisé, la reconstruction du passé s'accompagne de l'émergence de nouveaux acteurs dont la socialisation est fortement liée à l'identité affichée [Dubar, 2000]. À Pigüé, la redécouverte de ce passé français ou la construction d'un lien fort avec la France constitue une ressource importante au moment où les collectifs tentent de consolider cette forme de socialisation postmoderne.

Ainsi, pour ce second groupe d'acteurs, à l'inverse du premier, l'apprentissage de la langue française est essentiel. Ce n'est pas le cas de l'occitan dont on ne trouve plus guère de trace. Le français est le capital culturel essentiel pour bien se positionner dans le champ social local [Bourdieu, 1994] : la relation à la France ordonne le social. Il est remarquable de constater l'effort que font ces acteurs pour 
acquérir, par les études (et non par transmission intrafamiliale), un bon niveau de français. Aujourd'hui, les francophones de Pigüé se trouvent majoritairement dans ce deuxième groupe et non dans le premier. C'est ainsi que l'action la plus remarquable des acteurs du deuxième groupe est leur combat pour l'enseignement obligatoire du français en deuxième langue, dans les écoles primaires du district, privées et publiques. Le conseil scolaire du district de Saavedra, ainsi que l'Inspection de l'éducation de la province, ont accepté l'idée en tant que «projet pilote ». Ce fait, inédit en Argentine, perdure depuis 1998. Plus de 300 enfants entre 9 et 11 ans (de 5 écoles) suivent actuellement deux heures obligatoires de français par semaine. Le conseil général de l'Aveyron a financé la formation et la rémunération des cinq enseignants pendant les quatre premières années du projet. Le ministère argentin de l'Éducation a ensuite pris le relais.

Nous observons donc deux groupes d'acteurs qui se réclament de la France, mais aux stratégies divergentes sur le fond et la forme, sans toutefois se concurrencer. Certains de leurs interlocuteurs, comme l'ambassade de France en Argentine, entretiennent une position transversale, appuyant la coopération entre l'Aveyron et l'Amicale Pigüé et reconnaissant dans les visites et les actes protocolaires les origines et la représentation légitime de celles-ci par les acteurs du premier groupe. D'autre part, un troisième groupe insiste sur la dimension économique de la patrimonialisation. C'est notamment le cas de la nouvelle administration municipale péroniste de Pigüé, dont la cible électorale est les populations modestes. Historiquement, la mairie a fortement impulsé le lien avec l'Aveyron depuis les festivités du centenaire et les visites du président radical Raúl Alfonsín auprès des maires successifs, jusqu'alors tous issus des notables agricoles radicaux d'origine aveyronnaise. Il résulte de cette configuration un accès différencié à la culture française selon le niveau socioéconomique des habitants. Les habitants des quartiers populaires à faible condition économique, malgré l'enseignement du français à l'école, ont un accès relativement marginal aux actions de coopération interculturelle. Nos interlocuteurs du parti péroniste, au conseil municipal nous ont ainsi déclaré :

«La question de la culture française est restée plutôt élitiste, il faut populariser plus ».

Il apparaît ainsi que la nouvelle administration de la ville interprète le lien avec la France comme un projet des élites traditionnelles (groupe 1 d'acteurs) ou de nouvelles élites cultivées (groupe 2). Dès lors, les acteurs de la coopération franco-argentine estiment que la nouvelle administration municipale est moins active que les administrations antérieures. Cependant, son appui, certes plus modeste, n'a pas cessé, et poursuit la mise en patrimoine. Cette continuité, malgré l'alternance politique de la mairie, permet de maintenir le patrimoine français au sein de l'agenda public local [Muller, 2000]. Les membres du parti péroniste reconnaissent d'ailleurs le rôle positif joué par l'Amicale Pigüé dans la diffusion de la mémoire sociale qu'ils souhaitent par ailleurs rendre plus accessible aux classes populaires. Ils saluent en particulier l'enseignement du français dans les 
écoles primaires publiques, perçu comme un véritable atout pour les nouvelles générations. Par ailleurs, nos interlocuteurs ont une vision multiculturelle du patrimoine lorsqu'ils soulignent que la culture et l'histoire locale ont également été produites par d'autres «communautés », à savoir les immigrés espagnols (et notamment les Majorquins), les Italiens et les Allemands de la Volga.

Ce qui est intéressant chez les acteurs de la nouvelle administration municipale, c'est qu'ils lient explicitement ces aspects culturels à la dimension économique et à des bénéfices matériels pour le territoire. Selon eux, ce lien historique à la France, pour se légitimer pleinement et durablement, doit apporter un avantage économique au district.

\section{La bataille des traces : I'histoire locale en controverses}

Le processus de patrimonialisation et de construction de la mémoire collective n'est pas qu' un travail sur les souvenirs, il est aussi un travail physique, intellectuel (artistique notamment) et institutionnel de production de «traces » du passé dans le vécu des habitants d'aujourd'hui. Cette production de traces est en grande partie une légitimation, ce qui implique le déploiement de stratégies sociopolitiques et, parfois, des conflits d'interprétations et d'intérêts, voire des affrontements. Nous avons déjà parlé d'une trace immatérielle qui est celle de la langue, nous allons aborder à présent les traces matérielles qui peuvent être des objets, des événements et des produits, et bien souvent leur combinaison.

\section{Les livres et la légitimation sociale}

Le premier grand type de «traces » du passé français à Pigüé est incontestablement la production écrite et, en particulier, les livres. Cette production ne s'est pas confinée à des cercles érudits. Elle est connue des acteurs locaux qui, même s'ils n'ont pas forcément lu eux-mêmes tous ces ouvrages, en citent volontiers la référence pour légitimer leurs discours. La production de livres a été très importante depuis le centenaire, à tel point qu'il n'est pas possible ici de les citer tous, mais, par contre, nous pouvons en retenir un petit nombre qui a clairement marqué ce champ de pouvoir.

Nous avons déjà cité un ouvrage, essentiel à notre sens dans le processus de patrimonialisation, ayant pour auteurs des universitaires de Toulouse dans les années 1970 [Andreu et al., 1993]. Avant cette publication, il existait quelques essais sur la colonisation de Pigüé et le passé français, notamment l'un écrit par un grand éleveur local [Ducos, 1934] et l'autre par sous-officier de la marine [Monferrán Monferrán, 1955]. Mais le livre des universitaires français est le premier explicitement fondé sur des enquêtes auprès des habitants et proposant un inventaire exhaustif des familles. Il a ainsi donné un caractère public à ce qui était jusqu'alors une collection de récits privés. Ce livre a aussi préparé un processus d'identification collective dans des souvenirs et un territoire commun, et non dans un passé illustre et épique raconté par des notables. En ce sens, nous-mêmes 
auteurs de cet article et nos collègues universitaires de Toulouse avons de fait exercé une action patrimoniale à travers nos écrits et la formation universitaire. Nos recherches sur le développement local qui ont toujours inclus Pigüé, la réalisation d'un film de recherche [Fontorbes et al., 2013], la formation en doctorat à Toulouse ou à Bahía Blanca de divers jeunes de Pigüé, ont été un apport aux stratégies des acteurs de la coopération avec la France et, en particulier, avec l'Aveyron. Cet article même n'y échappe pas.

La coopération décentralisée avec l'Aveyron a ainsi fortement stimulé la production culturelle et les livres. L'ouvrage de l'équipe de Toulouse a été actualisé et réédité en 1993 (l'un d'entre nous a participé aux travaux de terrain), puis la région Midi-Pyrénées a financé une recherche sur Pigüé ouverte aux communautés voisines d'autres origines du sud-ouest de la province de Buenos Aires : juive, danoise, allemande de la Volga, etc., un projet de recherche que nous avons codirigé avec un collègue du CNRS [Tulet, Albaladejo, Bustos Cara, 2001]. Un livre bilingue, soutenu par la coopération franco-argentine, est consacré au peintre aveyronnais Numa Ayrinhac [Litre Valetin, 2009] et constitue un important complément au musée qui lui est dédié.

Les parents de Numa Ayrinhac se sont installés à Pigüé deux ans après l'arrivée du premier contingent de colons, quand Numa avait sept ans. Son père était maréchal-ferrant et sa mère couturière ; son unique frère était handicapé. Ses parents l'ont beaucoup encouragé à étudier à Bahía Blanca, puis à Buenos Aires où il apprend la peinture aux côtés de Ernesto de la Cárcova, et enfin, à Paris à l'école des Beaux-Arts. Il fut un peintre particulièrement prolifique, représentant des paysages de son Aveyron natal où il revint très régulièrement et de la pampa de Piguié. Ayrinhac est connu en Argentine pour ses peintures d'Éva Perón qui l'avait choisi comme peintre officiel, ce que sa famille, radicale, ne lui a jamais pardonné. Malgré ces œuvres si connues, leur auteur était tombé dans l'oubli tant en Argentine qu'en France et c'est la volonté de sa petite fille, sa passion pour son grandpère qui, à l'aide de la coopération avec l'Aveyron, a permis de publier cette biographie dans les deux pays, écrite par une étudiante de Pigüé.

Mais l'écrit le plus documenté est le journal de François Issaly dans lequel il a consigné quotidiennement tous les événements depuis son départ de la gare de Rodez, le 25 octobre 1884, avec le premier contingent, jusqu'à sa mort 50 ans plus tard et, plus précisément, deux mois avant les festivités du cinquantième anniversaire de la fondation de Pigüé. En plus de toute la correspondance avec Cabanettes qui a été intégralement conservée, le journal est un manuscrit de 5500 pages qui reste aujourd'hui entre les mains de sa petite fille Vita, une sociologue de la ville de La Plata qui, à sa retraite, est revenue vivre à Pigüé et a décidé d'exploiter ce document historique légué par sa mère afin, nous a-t-elle dit, de réhabiliter la mémoire de son grand-père.

Issaly a lié son destin à celui du premier contingent et s'est, lui aussi, installé sur un lot de terre. Il a vécu avec les premiers colons les désillusions et les souffrances d'une installation sur des terres vierges, mais aussi le conflit entre le 
spéculateur Casey et son entrepreneur de colonisation Aveyronnais, Cabanettes, qui terminera par l'expulsion de ce dernier. Casey nomma alors comme administrateurs deux colons français installés en seconde et troisième vague de colonisation et dont les descendants sont aujourd'hui de grandes familles terriennes locales. Un descendant de l'un d'entre eux, un Ducos, a d'ailleurs écrit le premier essai d'histoire locale dont nous avons parlé [Ducos, 1934]. La dureté de ces hommes et leurs pratiques violentes pour obtenir le recouvrement de la dette à Casey ont fractionné durablement la communauté française. Issaly a tout consigné dans son journal et le dénonçait de son vivant. Mais les administrateurs de Casey, qui connaissaient des juges influents à Buenos Aires, réussissent à le faire emprisonner pour une supposée pratique de la sorcellerie, justement l'année des cinquante ans de la fondation du village. Issaly meurt en prison deux mois avant les festivités organisées par la seconde génération de notables agraires français pour entretenir le souvenir, comme nous l'avons vu, et aussi pour l'orienter et en proposer une interprétation. À peine ce dernier décédé, le conseil municipal décida d'ériger un monument à la mémoire de Eduardo Casey en tant que «fondateur de la ville ». Un buste réalisé par Numa Ayrinhac fut ainsi opportunément inauguré à temps le 4 décembre 1934. Il est clair pour Vita que la commande avait été passée à Ayrinhac bien avant la mort de son grand-père, car on ne peut pas confectionner un buste en bronze en moins de deux mois, mais que cela avait été maintenu dans le secret jusqu'à ce que le seul érudit qui pouvait mettre à mal ce soudain statut de «fondateur de la ville » ne soit plus là pour parler et surtout pour écrire.

Au-delà de ces péripéties de l'histoire locale, que faut-il retenir ? Tout d'abord, qu'il ne s'agit pas d'anecdotes, mais de la construction d'une mémoire collective, et surtout de l'entrée des interprétations du passé dans l'espace public local. On peut enfin en débattre localement. De fait, le conseil municipal a étudié la demande de Vita et les documents qu'elle a produits et, fin 2015, a émis très officiellement un décret de réhabilitation de François Issaly en tant que cofondateur de la ville avec Clément Cabanettes. Il va sans dire que les acteurs gravitant autour de la Sociedad Francesa nous ont expliqué que, pour eux, Casey reste un fondateur injustement questionné.

Dans cette reconstruction des traces, l'énorme manuscrit de Issaly d'une valeur historique considérable est sorti de son statut d'objet privé, encore que le fait que l'unique sociologue qui l'analyse soit sa petite fille et que le lieu de conservation de l'original (sans aucune copie) soit toujours un domicile privé montre que sa « patrimonialisation » est loin d'être complète. Vita a en revanche permis que le conseil général de l'Aveyron scanne une petite partie de ce journal, et que celle-ci soit retranscrite et adaptée dans un livre édité en France dans sa langue originale [Issaly, 2014]. 


\section{Les monuments, musées et bâtiments : I'officialisation des personnages et des lieux du souvenir collectif}

Il va sans dire que l'histoire, non pas seulement de la colonisation, mais de son interprétation et de sa patrimonialisation, est inscrite dans le paysage et les objets urbains, par une sorte de sédimentation des discours officiels et des conflits. Au point culminant de l'énorme avenue centrale Casey, trône ainsi depuis 1925 la statue de Clément Cabanettes, qui est une œuvre impressionnante d'Ayrinhac, encore lui. Le buste de Casey a été ajouté, comme nous l'avons dit, sur une place latérale de l'avenue du même nom, et deux ans après le tournant du centenaire est apparu un médaillon de Issaly, sur une place de moindre importance quand même... Le musée Ayrinhac, celui de la colonisation mis en place par la municipalité, la station de chemin de fer parfaitement conservée depuis sa construction par les Anglais, les maisons de maître des riches familles aveyronnaises (certaines intactes avec leur mobilier d'origine), les œuvres d'art des tombeaux des familles françaises du cimetière, tout est sans aucun doute très intéressant, mais très peu exploité sur le plan touristique.

\section{L'alimentation et les fêtes : inventions et souvenirs}

Les entretiens avec tous nos interlocuteurs d'origine française nous ont révélé la consommation en famille de plats reconnus comme aveyronnais. C'est le cas selon eux de la «trufada », faite de fines tranches de pommes de terre cuites dans un peu d'huile avec de l'ail, du persil et du fromage (du Cantal en France). C'est aussi le cas du civet de lièvre, de cochonnailles comme le «fricando », un pâté en forme de boule enroulée dans un torchon, ou encore du cou d'oie farci et de diverses préparations à base de viande de canard. Mais tous ces aliments sont consommés dans un cadre exclusivement privé et, même si depuis les festivités du centenaire et surtout la coopération avec l'Aveyron ils sont identifiés par tous comme des traces du passé familial, ils ne sont en revanche pas mobilisés comme des facteurs d'identification collective.

Ce n'est plus le cas aujourd'hui d'un plat du nord de l'Aveyron qui s'appelle «l'estofinade », préparé avec de la morue, de la purée de pommes de terre et de l'huile de noix. Nos interlocuteurs se souviennent que leurs mères préparaient tous les ans au moins pour le Vendredi Saint ce plat avec le type de poisson (el cazón) et d'huile disponibles localement, et les acteurs du deuxième groupe que nous avons distingué ci-dessus n'ont découvert que récemment son origine. Le nom original est «stockfisch», car la morue était obtenue au Moyen-Âge des Anglais qui commerçaient depuis Bordeaux en remontant la vallée du Lot. Le mot a été transformé en « estofi », puis occitanisé en « estofinade ». Aujourd'hui, c'est le plat aveyronnais qui s'est le mieux conservé dans les familles depuis la colonisation et il est systématiquement reconnu et cité par tous en tant que patrimoine commun.

L'aligot à Pigüé est un cas intéressant d'une patrimonialisation réussie, bien que sans passé objectif, et il montre clairement que le patrimoine est une 
construction sociale, voire une invention, même si elle doit être en cohérence avec l'ensemble de la mémoire. Ce plat à base de purée de pommes de terre et de tomme fraîche est typique du nord-ouest de l'Aveyron et les acteurs argentins de la coopération l'ont découvert au cours de leurs voyages. On peut supposer que c'est un plat qui était connu des colons et qui a été oublié. Le fait est qu'aucun de nos enquêtés n'en avait le souvenir. Les voyageurs argentins, à la faveur des échanges de la coopération, ont rapporté chez eux des sachets d'aligot lyophilisé pour en partager l'expérience. Rapidement, ce plat inconnu prend de l'importance à Pigüé. Il est vrai que sa consommation est accompagnée systématiquement du fameux «chorizo » bien argentin, une saucisse de viande bovine (voire mixte ou de façon moins traditionnelle de viande de porc) partie intégrante dans toute l'Argentine de la grillade de viande (el asado). Très vite, l'aligot est élaboré à Pigüé avec différents fromages argentins, bien qu'aucun n'ait donné une réelle satisfaction face à la tomme de l'Aubrac. C'est alors qu'une jeune professeure du lycée agricole de Goyena effectue en avril 2009 un voyage d'études d'un mois au lycée agricole de Larroque (Rodez), sans parler français à ce moment-là. En lien avec la coopérative Jeune Montagne qui a mis en place l'AOC du fromage de Laguiole, autrement dit la tomme qui permet de faire l'aligot d'origine, elle passe quatre jours dans une ferme et parvient à son retour à faire fabriquer la tomme par son lycée. Plus tard, le lycée a importé des embryons de vaches Aubrac, l'une des deux races, avec la Simmental française, à être autorisées à fournir le lait du fromage AOC de Laguiole. Mais les naissances n'ont pas eu lieu et les initiateurs de cette opération se seraient découragés. Il faut comprendre dans le cas de l'importation de la race Aubrac et de l'aligot que la volonté des acteurs n'est pas celle d'un respect rigide de «l'authenticité » des traces de la colonisation et du passé, mais vise à exalter sans déformer une origine, et donc une originalité, par exemple avec une race bovine de format et d'aspect très différents des races courantes en Argentine et en particulier laitières.

Depuis 2009, l'Amicale Pigüé a inventé une fête annuelle de l'aligot le 25 octobre, le samedi le plus proche de la date anniversaire du départ de l'Aveyron du premier contingent. Une année sur deux, la fête est réservée aux élèves des écoles primaires et à leurs professeurs de français, avec plus de 300 participants. Les autres années, la fête est ouverte à toute la population, en prenant soin d'inviter officiellement des représentants des autres collectivités (espagnole, italienne, etc.). L'année précédant nos enquêtes en 2015, ce sont 600 personnes qui y ont participé. La moitié de l'aligot avait été confectionnée avec la tomme du lycée agricole de Goyena, et l'autre moitié avec de la tomme importée de la Coopérative Jeune Montagne. L'Amicale a fait de réels efforts pour aider les restaurateurs locaux à insérer l'aligot dans leurs cartes, comme inviter des chefs français de l'Aveyron et de Buenos Aires, etc., mais sans succès. Pourtant, l'aligot de Pigüé commence à être connu. En septembre 2015, l'Ambassade de France a demandé à l'Amicale de mettre en place, pour la journée de présentation des entreprises françaises à Buenos Aires, une dégustation d'aligot pour plus de 300 personnes. 
Mais un plat et une fête de Pigüé sont aussi très connus depuis 1999 : l'omelette géante de 40000 œufs produite le jour anniversaire de la fondation de Pigüé le 4 décembre (le premier dimanche du mois). La «Confrérie mondiale des chevaliers de l'omelette géante » est née en 1973 dans le village de Bessières en France, près de Montauban, sous une forme locale d'abord et rapidement elle a cherché à organiser ce type d'événement dans des lieux exotiques, intéressants et francophones : Nouvelle-Calédonie, Louisiane, Québec, et Pigüé. La «mondialisation » de ce réseau de joyeux notables locaux a été rendue possible, semble-t-il, par des opportunités de cooptation de nouveaux membres, ainsi que nos interlocuteurs nous l'ont présenté. Aujourd'hui, même si certains habitants se plaignent que leur fête locale soit un peu occultée par cette omelette, l'événement, connu dans tout le pays attire plus de 20000 personnes, et il profite fortement, même si brièvement, aux commerces locaux. Ce qui est intéressant, c'est que l'omelette est considérée en Argentine comme le plat national français, renforçant ainsi involontairement cette image de Pigüé au-delà de l'Aveyron. Également, cela permet d'observer que le groupe des acteurs traditionnels de la colonisation française n'est pas figé sur son passé ni sur l'Aveyron, et qu'il est capable d'une inventivité cohérente avec la stratégie identitaire et territoriale liée à la culture française.

Ainsi, aucune trace n'est totalement acquise et encore moins figée. Chacune demande un travail de maintien et de constante transformation. On voit aussi qu'aucune trace n'est le monopole d'un groupe d'acteurs, et que de nouveaux acteurs peuvent se les approprier légitimement et les mettre au profit de leurs stratégies, comme c'est en train de devenir le cas pour la maîtrise du français pour le deuxième groupe d'acteurs que nous avons distingué.

\section{Conclusion : de la patrimonialisation à la valorisation}

Depuis le centenaire de la fondation de la ville, nous avons identifié un processus de patrimonialisation du lien historique de Pigüé avec la France, à travers des efforts de réinterprétation et d'appropriation collective conduisant à une tendance à la publicisation de la relation à la culture française et à la territorialisation du sentiment d'appartenance. Les fêtes locales, l'enseignement obligatoire du français dans toutes les écoles primaires du district, les publications d'ouvrages et les musées ouverts à tous, l'inscription dans le paysage urbain de monuments et peintures murales et le début de production locale d'aliments relevant de ce passé, sont les éléments de cette publicisation et territorialisation. Aujourd'hui, plus personne dans le district n'ignore ce lien.

Mais à partir de quand le passé français devient-il le patrimoine de tous, autrement dit, à partir de quand ce processus de patrimonialisation s'inscrit-il dans la fabrication du sentiment d'être d'ici ? En ce qui concerne ce sens identitaire profond, le processus n'est sans doute pas aussi complet qu'il pourrait l'être, car il a du mal à aller au-delà des quartiers historiques en ville et des plus belles estancias (grandes exploitations) à la campagne. Cela ne doit pas occulter l'important travail 
de partage des références culturelles qui a été effectué depuis le centenaire, et le rôle essentiel de l'Amicale Pigüé d'une part et de la coopération avec l'Aveyron d'autre part. Ces acteurs ont eu une claire et efficace volonté d'ouverture sur le territoire et ils ont réussi, sans aucun doute, en peu d'années, à faire passer le lien avec la France d'un bien privé à un bien public. Ces acteurs nous montrent que la mise en patrimoine n'est pas passéiste ; à Pigüé elle a créé de nouveaux liens avec la France et l'Aveyron qui laisseront des traces pour au moins deux ou trois générations, et plus si le processus reste actif. Il est significatif que les francophones du district soient aujourd'hui plus le résultat de ces nouveaux liens qu'une rémanence du passé migratoire.

Mais pour les deux groupes d'acteurs que nous avons distingués, il y a bien une mise en patrimoine selon des stratégies radicalement différentes. Elle est évidente et même revendiquée explicitement comme telle pour le deuxième groupe autour de l'Amicale, car son intention est de faire partager le passé français avec tous les habitants du district quelles que soient leurs origines. De fait, l'enseignement du français et le programme de coopération se sont ouverts effectivement ou potentiellement à tous les habitants. Mais la mise en patrimoine au plan local n'est pas totalement absente du premier groupe centré sur la société française, dans la mesure où ces acteurs, à partir du cinquantième anniversaire de Pigüé, mettent en œuvre une visibilité locale à travers la mise en récit du passé, par les discours et les écrits ainsi que par les monuments, comme nous l'avons vu, même si les interprétations sur lesquelles est fondée cette mise en récits sont parfois vivement discutées dans l'espace public local. D'autre part, le cas de l'omelette géante montre leur capacité à faire largement rayonner le passé français de Pigüé et également à ne pas rester sur une interprétation figée ou passéiste. Finalement, ces deux groupes d'acteurs sont très complémentaires, et une des explications du succès de la patrimonialisation locale est, à notre sens, que ce ne sont pas les mêmes acteurs qui ont porté les stratégies familiale et territoriale.

La coopération avec le conseil général de l'Aveyron les a fortement soutenues. Mais il est clair que le processus ne peut dépendre éternellement des fonds de la coopération pour durer. En ce sens, la recherche d'un avantage économique de la part de l'actuelle administration municipale est salutaire. Cet avantage pourrait être touristique, comme le montre la haute fréquentation (encore que ponctuelle) due à l'omelette géante. Le potentiel est important, et ce serait le seul moyen de subvenir durablement, par exemple, à l'entretien d'un musée comme celui d'Ayrinhac. Mais il faudra une forte volonté politique du gouvernement local et la participation de certains acteurs socioéconomiques de Pigüé, notamment des autres collectivités culturelles qui ne pourront rester dans l'ombre et être écartées de cette valorisation. Lorsque l'on pense aujourd'hui à une possible valorisation touristique du passé français, on peut mesurer pleinement le chemin essentiel parcouru grâce aux acteurs locaux et à la coopération avec l'Aveyron : sans cette patrimonialisation locale, et sans cette appropriation active et authentique de la culture par les acteurs locaux passant par une territorialisation de celle-ci, la valorisation touristique pourrait difficilement aujourd'hui être autre chose qu'une 
« mise en tourisme », autrement dit, une folklorisation de la mémoire dans un but purement commercial à partir de simples réminiscences de la colonisation initiale (flèche 1 de la figure 3 ). À la suite du travail de construction d'une mémoire collective, la valorisation touristique a toutes les chances de faire partie d'un processus socio-économique plus large, plus stable, et surtout plus authentiquement, ancré dans les dynamiques socioculturelles locales (flèche 2 de la figure 3 ). Cette construction de la mémoire collective est certes un processus incessant, mais on peut se demander s'il est suffisamment avancé pour éloigner de possibles tentatives, de la part d'investisseurs extérieurs en particulier, de simple « mise en tourisme ». Malgré tout, il ressort de nos analyses qu'il est temps, voire urgent, que les politiques locales commencent cette valorisation économique et touristique, afin de ne pas décourager certains acteurs qui en dépendent, notamment ceux qui n'ont pas de passé français.

Figure 3 - Processus d'appropriation collective de la mémoire à Pigüé

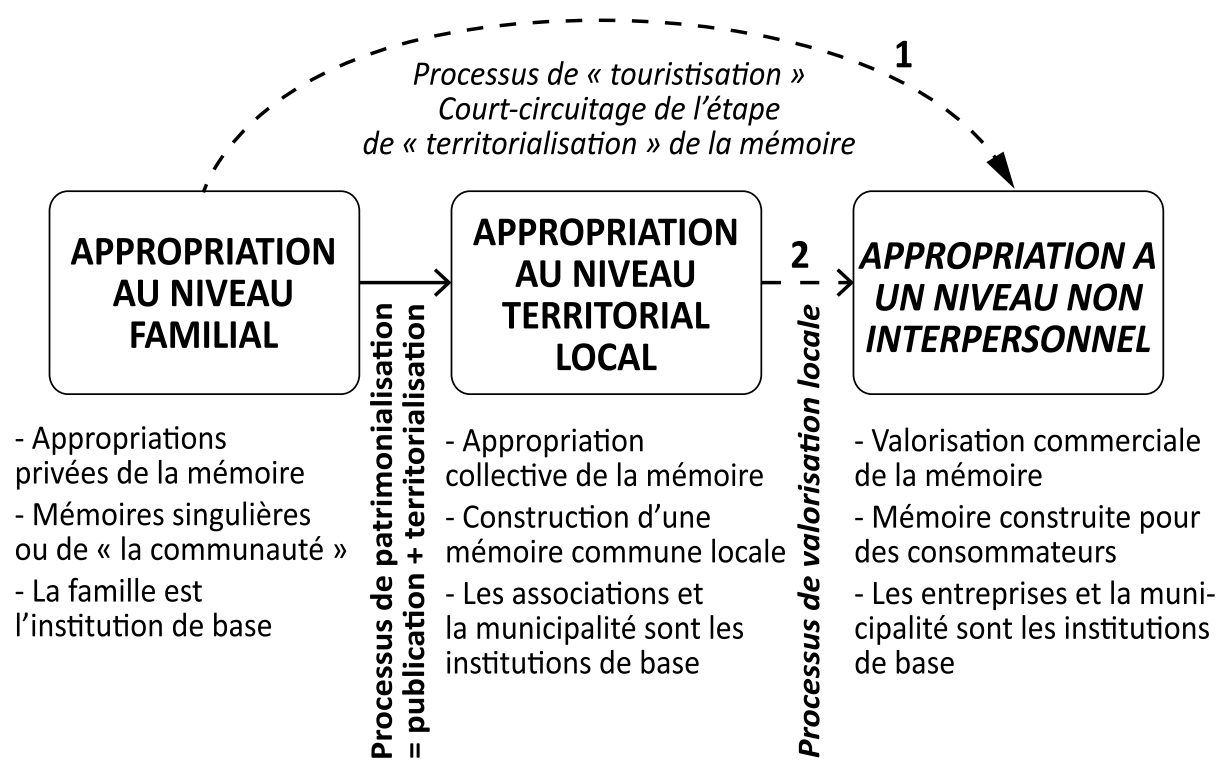

En italiques et pointillés les étapes 1 ou/et 2 qui ne sont pas (encore) produites à Pigüé

Source : élaboration par les auteurs.

\section{Bibliographie}

Andreu J., Bataillon C., Bennassar B., Gaignard R., Tulet J.-C. [1993], Les Aveyronnais dans la Pampa: fondation, développement et vie de la colonie aveyronnaise de Pigüé Argentine, 1884-1992, Toulouse, Privat, Presses universitaires du Mirail.

Baussant M. [2007], «Penser les mémoires », Ethnologie française, vol. 37, nº 3, p. 389-394. 
Baussant M., Dos Santos I., Ribert E., Rivoal I. [2015], « Logiques mémorielles et temporalités migratoires. Une introduction », in Baussant M., Dos Santos I., Ribert E., Rivoal I. (dir.), Migrations humaines et mises en récit mémorielles. Approches croisées d'anthropologie et de préhistoire, Paris, Presses universitaires de Paris Ouest, Éditions de la MAE, p. 11-33.

Bourdieu P. [1994], Raisons pratiques. Sur la théorie de l'action, Paris, Seuil.

Demazière D., Dubar C. [1997], Analyser les entretiens biographiques. L'exemple des récits d'insertion, Paris, Nathan.

Devoto F. [2003], Historia de la inmigración en la Argentina, Buenos Aires, Editorial Sudamericana.

Di Liscia M. H., B., Lassalle A. M. [2002], «Esta fue mi vida. No se la deseo a ninguna ». A propósito de «Narración de mi vida, 1884-1937», de Anaïs Vialka, Santa Rosa, Universidad nacional de La Pampa.

DubAR C. [2000], La crise des identités. L'interprétation d'une mutation, Paris, Presses universitaires de France.

Ducos O. F. [1934], Cincuentenario de la colonia francesa de Pigüé. Reseña histórica por sus vecinos, Pigüé, provincia de Buenos Aires.

Fontorbes J.-P., Granié A.-M., Lorda A., Bustos Cara R., Albaladejo C. [2013], El campo, el barrio, otras maneras de vivir en Pigüé, film de 50 minutes, ANR et AIRD (producteurs), Toulouse, France, ENFA.

Gaignard R. [1989], La Pampa argentina. Occupación - poblamiento - explotación, de la conquista a la crisis mundial (1550-1930), Buenos Aires, Solar.

Halbwachs M. [1925], Les cadres sociaux de la mémoire, Paris, Albin Michel.

Halbwachs M. [1950], La mémoire collective, Paris, Albin Michel.

Issaly F. [2014], De l'Aveyron à l'Argentine : itinéraire d'un pionnier, chronique de la fondation de la colonie française aveyronnaise de Pigüéld'après le manuscrit de François Issaly, 1924, adaptation de Nadine Costes, avec la collaboration de Louis Costes et Eduardo Varni, Rodez, Archives départementales de l'Aveyron.

LAVABre M.-C. [2000], «Usages et mésusages de la notion de mémoire », Critique internationale, $\mathrm{n}^{\circ}$ 7, p. 48-57.

Lavabre M.-C. [2007], «Paradigmes de la mémoire », Transcontinentales, vol. 5, $\mathrm{n}^{\circ} 9$, p. 139-147.

Litre Valetin M. L. [2009], Numa Ayrinhac. De la France à la Pampa, Buenos Aires, Editorial Dunken.

MONFERRÁn E. E. [1955], Fundación de la colonia francesa y pueblo de Pigüé (4 de diciembre de 1884), Buenos Aires, Cadel.

Muller P. [2000], Les politiques publiques, Paris, Presses universitaires de France.

Otero H. [2012], Historia de los Franceses en la Argentina, Buenos Aires, Editorial Biblos.

REy BALMACEDA R. C. [1994], «Bibliografía sobre Inmigración, colonización y comunidades estranjeras en la Argentina », Geodemos, n 3, p. 1-302.

Samson C., Becouez V. [2013], Aveyron Pigüé, les descendants, Lascelle, Éditions de la Flandonnière.

Tulet J.-C., Albaladejo C., Bustos Cara R. [2001], Une Pampa en mosä̈que. Des communautés locales à l'épreuve de l'ajustement en Argentine, Paris, L'Harmattan. 